\title{
Author Correction to: Born-digital archives
}

\author{
Thorsten Ries ${ }^{1,2} \cdot$ Gábor Palkó $^{3}$ \\ Published online: 16 September 2019 \\ (C) Springer Nature Switzerland AG 2019
}

\section{Author Correction to: International Journal of Digital Humanities (2019) 1:1-11 https://doi.org/10.1007/s42803-019-00011-x}

The original version of this article unwittingly omitted the due and necessary acknowledgment, which is hereby added below:

The research for this work and editing of the special issue conducted by Thorsten Ries has been funded by the Marie-Sklodowska-Curie Fellowship DFitHH - Digital Forensics in the Historical Humanities and the Research Foundation - Flanders, FWO.

The online version of the original article can be found at https://doi.org/10.1007/s42803-019-00011-x

Thorsten Ries

Thorsten.Ries@ugent.be; T.Ries@sussex.ac.uk

$\triangle$ Gábor Palkó

palko.gabor@btk.elte.hu

1 Ghent University, Ghent, Belgium

2 University of Sussex, Brighton, UK

3 Eötvös Loránd University, Budapest, Hungary 